\title{
Repeat Administration of Reversal Agents in \\ Patients Receiving Neostigmine or Sugammadex: A Retrospective Observational Study
}

Laura Gilbertson ( $\square$ laura.gilbertson@emory.edu )

Emory University Alzheimer's Disease Research Center https://orcid.org/0000-0001-5501-8943

Francis Wolf

Emory University School of Medicine

Grant C. Lynde

Emory University School of Medicine

Research article

Keywords: residual neuromuscular blockade, reversal, sugammadex

Posted Date: March 21st, 2020

DOI: https://doi.org/10.21203/rs.3.rs-18193/v1

License: (c) (i) This work is licensed under a Creative Commons Attribution 4.0 International License.

Read Full License 


\section{Abstract}

Background: Antagonism of neuromuscular blockade (NMB) induced by rocuronium and vecuronium can be achieved with either neostigmine or sugammadex. Compared to sugammadex, antagonism with neostigmine is more likely to result in incomplete reversal, i.e. residual neuromuscular blockade (rNMB). The administration of additional doses of a reversal agent following an initial reversal dose may be a marker for suspected rNMB. We studied the frequency and temporal patterns of repeat (rescue) administration of reversal agents in patients who received an initial dose of neostigmine vs. sugammadex.

Methods: We analyzed retrospective data from electronic anesthesia records to identify surgical patients who received rescue dose reversal, defined as two or more doses of reversal agent, following administration of non-depolarizing NMB and extubation in the operating room. We assessed rates of rescue reversal over time following the introduction of sugammadex and compared rescue rates for patients receiving neostigmine vs sugammadex.

Results: A total of 24,027 cases using cisatracurium, rocuronium, and vecuronium were analyzed. Following the addition of sugammadex to formulary in 2016, reversal with neostigmine decreased from $79 \%$ to $5.3 \%(p<0.001)$ and the use of rescue reversal after neostigmine increased from $6.0 \%$ to $18 \%$ $(p<0.001)$. In contrast, rescue reversal after sugammadex was $2.5 \%$, with no change over the study period $(p=0.059)$. The percentage of patients who were not given any reversal agent following non-depolarizing NMB decreased from $20 \%$ to $13 \%(p<0.001)$. As neostigmine usage progressively decreased after introduction of sugammadex, there was a corresponding increase in rescue reversal dosing when initial reversal was attempted with neostigmine.

Conclusions: Repeated administration of a reversal agent was 7 times more likely to occur in patients initially reversed with neostigmine compared to sugammadex. This finding likely reflects increased rates of observed weakness in patients reversed with neostigmine. The correlation between decreasing neostigmine use and increasing rescue reversal after neostigmine may indicate a progressive decline in the effective use of neostigmine (as knowledge and experience waned), and/or decreasing confidence in its efficacy.

\section{Background:}

Until 2016, anesthesia providers in the United States relied solely on anticholinesterase inhibitors (mainly neostigmine) to antagonize the effects of non-depolarizing neuromuscular blocking drugs (NMBDs). Safe and effective use of NMBDs relying on full antagonism with neostigmine can be challenging since neostigmine is not a true reversal agent but an indirect antagonist that is ineffective for reversing from deep or profound block(1) and has unpredictable results at moderate and even shallow blockade. $(2,3)$ Full reversal of neuromuscular block with neostigmine requires careful dosing of NMBDs, proper monitoring of blockade, and the presence of both sufficient spontaneous recovery prior to, and sufficient 
time following, administration.(4-6) As a result, about $40 \%$ of patients have residual neuromuscular blockade (rNMB) in the recovery room following reversal with neostigmine.(4)

With the introduction of sugammadex to the US in 2006, rapid reversal from deep, and even profound neuromuscular block was possible using relatively simple dosing.(7-10) Studies have suggested that rNMB can be eliminated with the use of sugammadex, if dosed correctly based on twitch monitoring at the ulnar nerve.(11) At our institution, sugammadex was made available without restriction for use by anesthesia providers and in less than a year it became the reversal agent of choice, used in $>75 \%$ of cases with non-depolarizing NMBDs. In previous work,(12) we showed that the introduction of sugammadex was associated with a reduction in severe cases of $\mathrm{rNMB}$, as evidenced by fewer patients requiring mechanical ventilation in the post-anesthesia recovery unit (PACU). We had also informally observed that sugammadex was being used in a "rescue" fashion to reverse suspected rNMB in patients who appeared clinically weak following an initial dose of neostigmine.

Although administration after incomplete reversal with neostigmine is not an approved indication for sugammadex, case reports have highlighted the potential use of sugammadex to treat rNMB after incomplete reversal with neostigmine $(13,14)$ and others have suggested that administering neostigmine combined with a subsequent lower dose of sugammadex could be an effective and cost-saving strategy to reversal.(15)

In the current work, we studied the use of sugammadex as a rescue reversal among surgical patients at a tertiary care academic medical center. We sought to determine the frequency of sugammadex rescue in patients who had been reversed with neostigmine compared to those who had been reversed with sugammadex.

\section{Methods:}

This study was approved by the Emory University Institutional Review Board (IRB00109451) prior to data acquisition and analysis, including a waiver of written informed consent. Our data analysis plan was included with our IRB submission. This manuscript was prepared in accordance with the Strengthening the Reporting of Observational Studies in Epidemiology (STROBE) statement and guidelines for improved reporting of observational studies and propensity score analyses.(16)

\section{Setting}

Emory University Hospital is a large academic medical center with cases being performed using a care team model of an Anesthesiologist supervising a resident, Anesthesia Assistant, or Certified Registered Nurse Anesthetist. Institutional policy requires the use of a qualitative nerve stimulator prior to patient extubation. Additionally, institutional practice is to wait for the presence of at least 1 out of 4 twitches on train-of-four assessment prior to the administration of neostigmine. Sugammadex was approved by the Emory University Pharmacy and Therapeutics committee beginning April 2016 and made available for use in the operating rooms without restriction. 


\section{Inclusion Criteria}

All cases performed in the main operating rooms at Emory University Hospital utilizing the electronic medical record between January 2016 and December 2018 who were administered cisatricurium, rocuronium, or vecuronium were included in this analysis.

\section{Analysis}

We analyzed retrospective data from electronic anesthesia records as stored in our institutional database to identify surgical patients who received rescue dose reversal (defined as two or more doses of reversal agent) following non-depolarizing neuromuscular blockade (defined as any dose of rocuronium, vecuronium, or cisatracurium). We assessed reversal and rescue rates over time following the introduction of sugammadex and compared rescue rates for patients receiving neostigmine vs sugammadex.

\section{Statistical Analysis}

Statistical analysis was performed using JMP® Pro version 13.0.0 (SAS Institute Inc., Cary, NC, 19892019). Student's t-test was used to compare demographic differences between patient ages, sex, and use of neuromuscular blockade reversal over time. Regression analysis using fit least squares was used to evaluate frequency of additional doses of neuromuscular blockade reversal as rates as compared to overall utilization of neostigmine as the primary reversal agent.

\section{Results:}

A total of 24,027 cases using cisatricurium, rocuronium, and vecuronium were performed in this time interval. Neostigmine was utilized in 4,327 cases, sugammadex in 16,016, and no reversal was used in 3,684 cases. Following the introduction of sugammadex to formulary, the frequency of neostigmine reversal and patients receiving no reversal both decreased in a statistically significant manner $(p<0.001$ for each) (Fig. 1). In 776 cases, two or more doses of neuromuscular blockade reversal were administered to a patient (i.e. rescue reversal cases). Prior to the introduction of sugammadex, $6.0 \%$ of cases utilizing neostigmine for reversal received rescue doses (all with neostigmine), however by $2018,18 \%$ of cases utilizing neostigmine received additional doses of NMB reversal ( $p<0.001$ ) (Fig. 2). In contrast, only $2.5 \%$ of patients reversed with sugammadex required additional reversal agent, and this did not increase over time $(p=0.059)$. Of the 251 cases in 2018 that received two or more doses, of NMB reversal, 37 used neostigmine initially followed by sugammadex.

\section{Discussion:}

In this study, rescue reversal after neostigmine was 7 times higher than rescue reversal after sugammadex, and the majority of patients who received rescue reversal were given sugammadex rather than neostigmine. This highlights the greater effectiveness and providers' growing confidence in sugammadex as a superior reversal agent. 
We also observed that the overall proportion of patients given additional reversal after initial neostigmine reversal significantly increased following the addition of sugammadex to formulary. This is demonstrated by the correlation between decreased neostigmine usage and increased rescue rates following neostigmine.

After its introduction into clinical use at our facility, sugammadex has rapidly gained popularity as the primary means of neuromuscular blockade reversal in the operating room owing to its ease of dosing and rapid reversal. As sugammadex reversal has become increasingly common, the total number of patients receiving neostigmine reversal has significantly declined. With this regression in neostigmine reversal, we observed a 3-fold increase in the prevalence of patients given rescue reversal after attempted reversal with neostigmine (6\% vs $18 \%)$.

In part our findings illustrate the reduced likelihood of rNMB when reversing with sugammadex. AbadGurumeta et al. performed a systematic review showing that sugammadex reduced all signs of residual postoperative paralysis compared with neostigmine, with a relative risk reduction of at least $50 \%$ (17). A recent Cochrane review comparing sugammadex and neostigmine reversal also showed meaningfully reduced signs of postoperative residual paralysis in the sugammadex group with a relative risk of 0.40(18). O'Reilly-Shah et al. demonstrated the clinical implications of this by showing that the introduction of sugammadex at a large tertiary hospital resulted in a significant decrease in the incidence of mechanical ventilation in PACU due to reduced incidence of $\mathrm{rNMB}(12)$.

While the evidence is convincing that patients who receive sugammadex reversal have less residual neuromuscular blockade, it is noteworthy that patients who are now receiving neostigmine reversal have an even higher prevalence of rescue reversal than previously seen(19). This phenomenon is no doubt due in part to the fact that sugammadex actually allows for reversal of residual blockade in cases where additional doses of neostigmine would not have been effective due to neostigmine's ceiling effect, which precludes any further positive effects on recovery once a full dose has been given. However, other explanations may be at play, as this does not fully justify the rising use of rescue after initial reversal with neostigmine.

There are several possible explanations for this finding. One possibility is that providers are becoming less and less familiar with neostigmine dosing and effective usage, due to the increased preference for sugammadex reversal (many anesthesia residents remain unfamiliar with neostigmine dosing due to prevalence of sugammadex reversal). Alternatively, providers may have become more perceptive in recognizing residual weakness after seeing the rapid return to full strength with administration of sugammadex. Regardless of the reason, it is important for providers to be cognizant that there have been increased episodes of suspected residual weakness in patients receiving neostigmine. Those providers choosing to use neostigmine reversal must be meticulous in assessing neuromuscular blockade when dosing and confirming full reversal(20). Use of a quantitative train of four monitor is recommended for safe reversal with neostigmine(21). 


\section{Study Limitations}

While this study was conducted at a large academic hospital, it was performed at a single hospital, which may limit the patient diversity and provider practice variability and therefore ability to generalize the results to other institutions.

We looked at repeat dosing of reversal agents and think that is likely a marker for cases of suspected weakness based on provider assessment. One potential weakness with our definition of rescue reversal is that providers might choose to give reversal in divided doses to allow for more gradual reversal and assessment of response. We think this phenomenon had limited impact on our data as the usual practice in our institution is to document the full dose and add a comment to note if it was given in divided doses. Also, we did not measure rNMB directly using a quantitative nerve monitor, nor did we prospectively observe practice, so our interpretation of the reason for rescue dosing (suspected rNMB) is necessarily somewhat speculative. Our institution primarily utilizes qualitative train-of-four monitoring (TOF) as opposed to quantitative measurements (which are available but not routinely used). While quantitative TOF measurement has been shown to be superior to qualitative, there is still a substantial proportion of anesthesia providers using this measurement.

The majority of patients who received a rescue dose of reversal did not have a TOF assessment documented at the time of rescue administration. Rather, the decision to administer a second dose of reversal agent was made by the anesthesiologist based on subjective clinical features such as tachypnea, low tidal volumes, shortness of breath and upper airway obstruction suggestive of residual neuromuscular blockade.

\section{Conclusion:}

Safe and full reversal of NMB with neostigmine is challenging even when used regularly; with irregular use the rates of weakness may be further increased. Unless they want to abandon the use of neostigmine altogether, anesthesia providers need to stay vigilant with NMB management and neostigmine reversal, minimize the dose of NMB when suitable, dose reversal appropriately and early enough, and ensure proper assessment of recovery, ideally with quantitative monitoring. Those in academic institutions also need to continue to provide education on the correct reversal techniques with both sugammadex and neostigmine so that future providers are proficient in the use of both methods of reversal. Further studies evaluating the use and effectiveness of sugammadex as the treatment for residual neuromuscular blockade in the PACU need to be performed.

\section{Abbreviations}

1. $\mathrm{NMB}=$ neuromuscular blockade

2. $\mathrm{rNMB}=$ residual neuromuscular blockade

3. NMBDs= neuromuscular blocking drugs 
4. $\mathrm{PACU}=$ post-anesthesia recovery unit

5. TOF= train-of-four

\section{Declarations}

Ethics Approval: This study was approved by the Emory University Institutional Review Board (IRB00109451) prior to data acquisition and analysis, including a waiver of written informed consent. Our data analysis plan was included with our IRB submission.

Consent for publication: Not applicable

Availability of data and materials: The datasets generated and/or analyzed during the current study are not publicly available due to potential of compromising individual privacy but are available from the corresponding author on reasonable request.

Competing Interests: Grant C Lynde receives consulting fees from Merck Pharmaceuticals. No other authors have conflicts of interest.

Funding: This research received no specific grant from any funding agency in the public, commercial, or not-for-profit sectors. The Emory University Department of Anesthesiology generously supported the time of the authors in producing this work.

\section{Author Contributions:}

LG interpreted the data, drafted and critically revised the manuscript. FW interpreted the data, drafted and critically revised the manuscript. GL conceived the work, acquired the data, interpreted the data, drafted and critically revised the manuscript. All authors read and approved the final manuscript.

Acknowledgements: Lisa Cogdill, MPH

\section{References}

1.

Jones RK, Caldwell JE, Brull SJ, Soto RG. Reversal of profound rocuronium-induced blockade with sugammadex: a randomized comparison with neostigmine. Anesthesiology. 2008;109(5):816-24.

2.

Blobner M, Eriksson LI, Scholz J, Motsch J, Della Rocca G, Prins ME. Reversal of rocuronium-induced neuromuscular blockade with sugammadex compared with neostigmine during sevoflurane anaesthesia: results of a randomised, controlled trial. Eur J Anaesthesiol. 2010;27(10):874-81.

3.

Brull SJ, Murphy GS. Residual neuromuscular block: lessons unlearned. Part II: methods to reduce the risk of residual weakness. Anesth Analg. 2010;111(1):129-40.

4. 
Murphy GS, Brull SJ. Residual neuromuscular block: lessons unlearned. Part I: definitions, incidence, and adverse physiologic effects of residual neuromuscular block. Anesth Analg. 2010;111(1):120-8. 5.

Brull SJ, Kopman AF. Current Status of Neuromuscular Reversal and Monitoring: Challenges and Opportunities. Anesthesiology. 2017;126(1):173-90.

6.

Maybauer DM, Geldner G, Blobner M, Puhringer F, Hofmockel R, Rex C, et al. Incidence and duration of residual paralysis at the end of surgery after multiple administrations of cisatracurium and rocuronium. Anaesthesia. 2007;62(1):12-7.

7.

Hristovska AM, Duch P, Allingstrup M, Afshari A. Efficacy and safety of sugammadex versus neostigmine in reversing neuromuscular blockade in adults. Cochrane Database Syst Rev. 2017;8:CD012763. 8.

Naguib M. Sugammadex: another milestone in clinical neuromuscular pharmacology. Anesth Analg. 2007;104(3):575-81.

9.

Lee C, Jahr JS, Candiotti KA, Warriner B, Zornow MH, Naguib M. Reversal of profound neuromuscular block by sugammadex administered three minutes after rocuronium: a comparison with spontaneous recovery from succinylcholine. Anesthesiology. 2009;110(5):1020-5.

10.

Abrishami A, Ho J, Wong J, Yin L, Chung F. Sugammadex, a selective reversal medication for preventing postoperative residual neuromuscular blockade. Cochrane Database Syst Rev. 2009(4):CD007362.

11.

Brueckmann B, Sasaki N, Grobara P, Li MK, Woo T, de Bie J, et al. Effects of sugammadex on incidence of postoperative residual neuromuscular blockade: a randomized, controlled study. $\mathrm{Br} \mathrm{J}$ Anaesth. 2015;115(5):743-51.

12.

O'Reilly-Shah VN, Lynde GC, Mitchell ML, Maffeo CL, Jabaley CS, Wolf FA. Initial experience with the unrestricted introduction of sugammadex at a large academic medical center: a retrospective observational study examining postoperative mechanical ventilation and efficiency outcomes. Korean $\mathrm{J}$ Anesthesiol. 2018;71(5):374-85.

13.

Ledowski T, Falke L, Johnston F, Gillies E, Greenaway M, De Mel A, et al. Retrospective investigation of postoperative outcome after reversal of residual neuromuscular blockade: sugammadex, neostigmine or no reversal. Eur J Anaesthesiol. 2014;31(8):423-9.

14.

Green MS, Venkatesh AG, Venkataramani R. Management of Residual Neuromuscular Blockade Recovery: Age-Old Problem with a New Solution. Case Rep Anesthesiol. 2017;2017:8197035. 15. 
Aouad MT, Alfahel WS, Kaddoum RN, Siddik-Sayyid SM. Half dose sugammadex combined with neostigmine is non-inferior to full dose sugammadex for reversal of rocuronium-induced deep neuromuscular blockade: a cost-saving strategy. BMC Anesthesiol. 2017;17(1):57.

16.

Von Elm E, Altman DG, Egger M, Pocock SJ, Gøtzsche PC, Vandenbroucke JP, et al. The Strengthening the Reporting of Observational Studies in Epidemiology (STROBE) Statement: guidelines for reporting observational studies. 2014;12(12):1495-9.

17.

Abad-Gurumeta A, Ripolles-Melchor J, Casans-Frances R, Espinosa A, Martinez-Hurtado E, FernandezPerez C, et al. A systematic review of sugammadex vs neostigmine for reversal of neuromuscular blockade. Anaesthesia. 2015;70(12):1441-52.

18.

Hristovska AM, Duch P, Allingstrup M, Afshari A. The comparative efficacy and safety of sugammadex and neostigmine in reversing neuromuscular blockade in adults. A Cochrane systematic review with meta-analysis and trial sequential analysis. Anaesthesia. 2018;73(5):631-41.

19.

Olesnicky BL, Traill C, Marroquin-Harris FB. The effect of routine availability of sugammadex on postoperative respiratory complications: a historical cohort study. Minerva Anestesiol. 2017;83(3):24854.

20.

El-Orbany M, Ali HH, Baraka A, Salem MR. Residual neuromuscular block should, and can, be a "never event". Anesth Analg. 2014;118(3):691.

21.

Checketts MR, Alladi R, Ferguson K, Gemmell L, Handy JM, Klein AA, et al. Recommendations for standards of monitoring during anaesthesia and recovery 2015: Association of Anaesthetists of Great Britain and Ireland. Anaesthesia. 2016;71(1):85-93.

\section{Tables}

Table 1. Patient characteristics. 2016 is the referent for tests of statistical significance. 


\begin{tabular}{|c|c|c|c|c|c|c|}
\hline & & $\underline{2016}$ & $\underline{2017}$ & $\underline{p \text { value }}$ & $\underline{2018}$ & p value \\
\hline Patient Age range (mean) & & $13-95(54.2)$ & $13-94(54.5)$ & 0.300 & $11-97(54.6)$ & 0.193 \\
\hline \multirow{2}{*}{ Sex } & Male & 3604 & 3650 & 0.575 & 3835 & 0.586 \\
\hline & Female & 4144 & 4273 & & 4521 & \\
\hline \multicolumn{2}{|c|}{ Total Cases Using Neuromuscular Blockade } & 7,748 & 7,923 & 0.077 & 8,356 & 0.013 \\
\hline & Neostigmine & 3,003 & 880 & 0.003 & 444 & $<0.001$ \\
\hline & Sugammadex & 3,287 & 5,909 & $<0.001$ & 6,820 & $<0.001$ \\
\hline & No Reversal & 1,458 & 1,134 & $<0.001$ & 1,092 & $<0.001$ \\
\hline \multicolumn{2}{|c|}{ Cases requiring $\geq 2$ reversal doses } & 282 & 243 & 0.282 & 251 & 0.227 \\
\hline \multicolumn{2}{|c|}{$\begin{array}{r}\text { Cases initially reversed with neostigmine requiring } \geq 2 \text { reversal } \\
\text { doses } \%(n)\end{array}$} & $7.2 \%(217)$ & $12.2 \%(107)$ & 0.006 & $18.0 \%(80)$ & $<0.001$ \\
\hline \multicolumn{2}{|c|}{$\begin{array}{r}\text { Cases initially reversed with sugammadex requiring } \geq 2 \text { reversal } \\
\text { doses } \%(n)\end{array}$} & $2.0 \%(65)$ & $2.3 \%(136)$ & 0.312 & $2.5 \%(171)$ & 0.059 \\
\hline
\end{tabular}

Figures

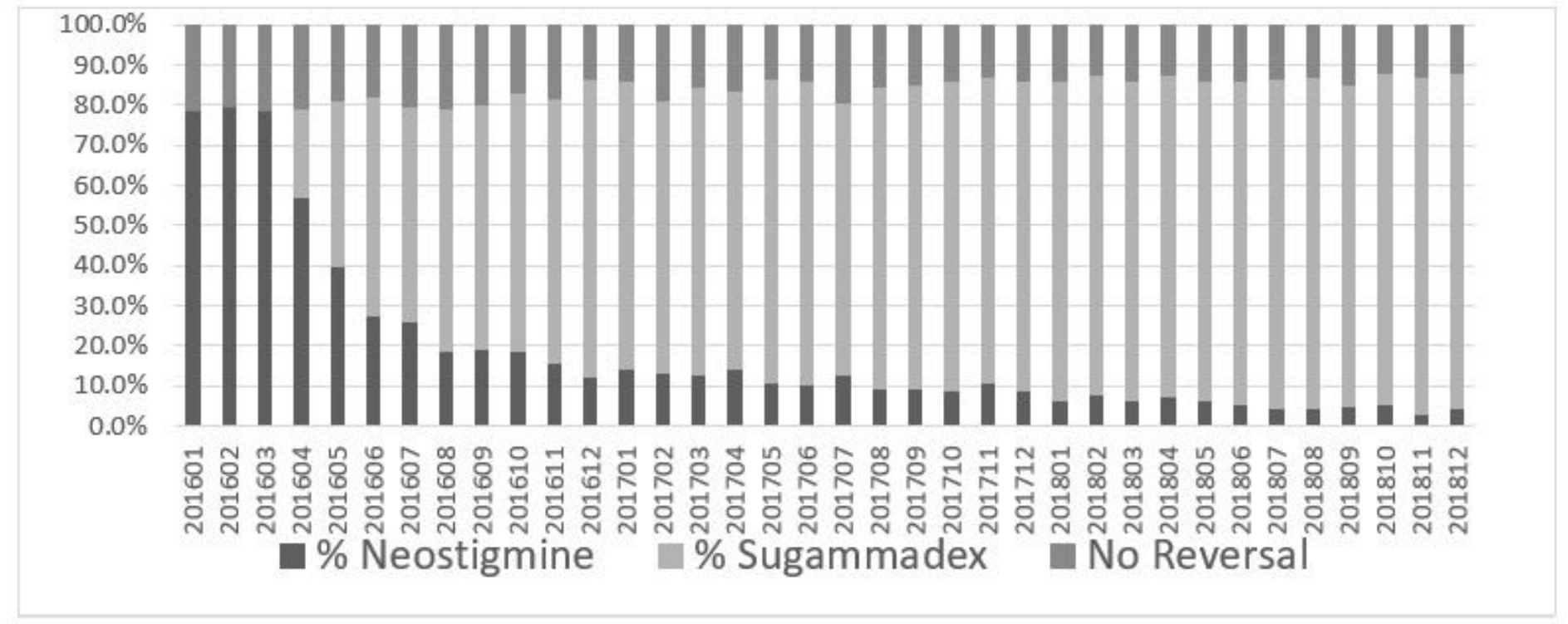

Figure 1 
Rates of neuromuscular blockade reversal with neostigmine, sugammadex, and no reversal over time.

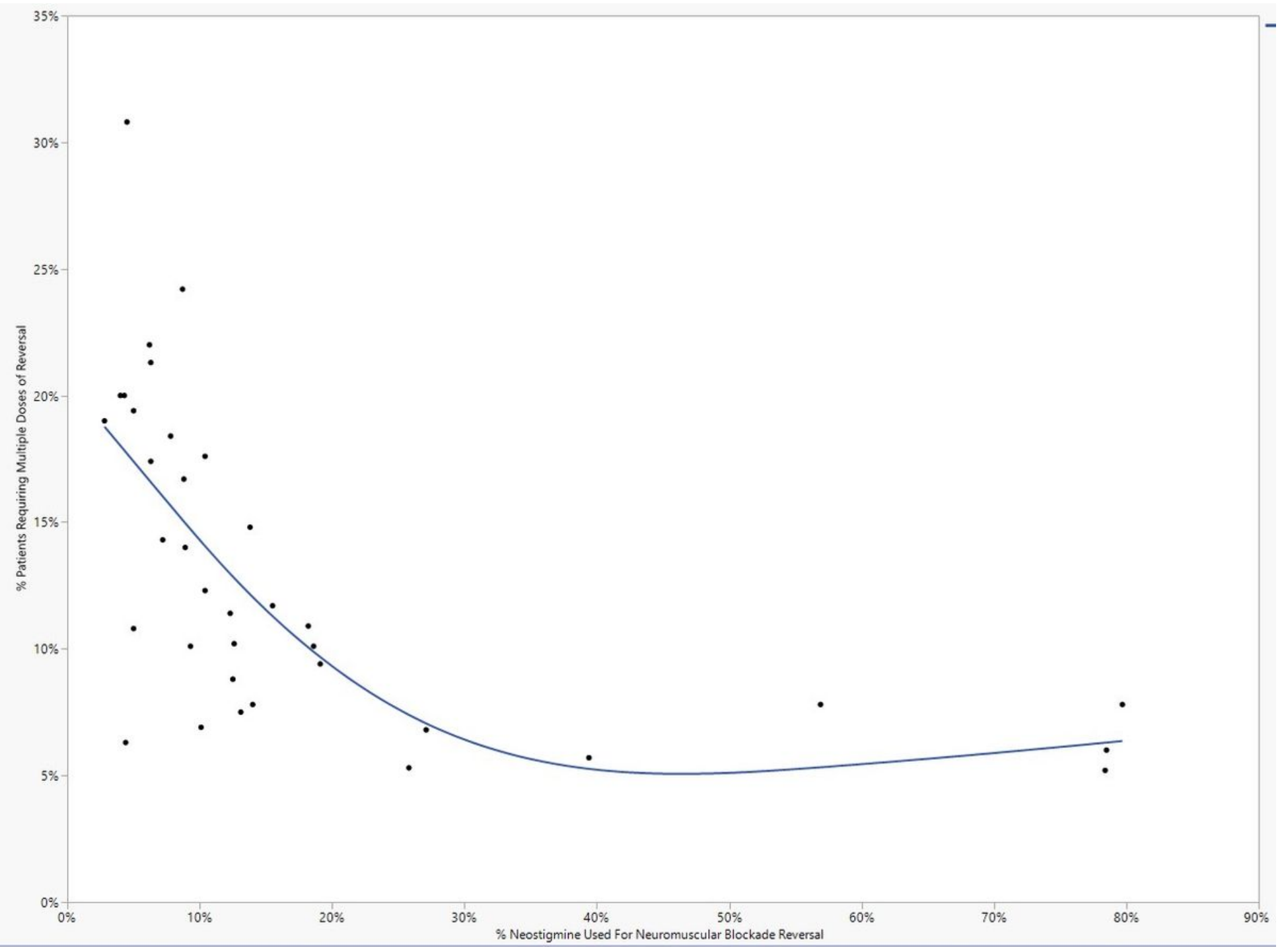

\section{Figure 2}

Frequency of neuromuscular blockade rescue reversal graphed against neostigmine use 